\title{
Madelung's disease - progressive, excessive, and symmetrical deposition of adipose tissue in the subcutaneous layer: case report and literature review
}

This article was published in the following Dove Press journal:

Diabetes, Metabolic Syndrome and Obesity:Targets and Therapy

\author{
Monika Szewc' \\ Robert Sitarz ${ }^{1-3}$ \\ Nina Moroz ${ }^{2}$ \\ Ryszard Maciejewski ${ }^{1, *}$ \\ Ryszard Wierzbicki ${ }^{2,4, *}$ \\ 'Department of Human Anatomy, \\ Medical University of Lublin, Lublin, \\ Poland; ${ }^{2}$ Department of Surgery with \\ Trauma, Orthopaedic and Urological \\ Subunit, Independent Public Health \\ Care Center of the Ministry of \\ Interior and Administration in \\ Lublin, Lublin, Poland; ${ }^{3}$ Department \\ of Surgery, St John's Cancer Center, \\ Lublin, Poland; ${ }^{4}$ Department of \\ Surgical Oncology, Medical University \\ of Lublin, Lublin, Poland
}

*These authors contributed equally to this work
Correspondence: Ryszard Wierzbicki Department of Surgery with Trauma, Orthopaedic and Urological Subunit, Independent Public Health Care Center of the Ministry of Interior and Administration in Lublin, Grenadierów Street 3, 20-33I Lublin, Poland

Tel +48817284258

Email skalpel@eu.pl

\begin{abstract}
Madelung's disease is a rare disorder described for the first time in the year 1846 by Brodie. It is characterized by the occurrence of progressive, excessive, and symmetrical deposits of adipose tissue in the subcutaneous layer. Most often, these changes concern the neck, the nape of the neck, arms, and upper back, giving the patients a specific, pseudoathletic appearance. Madelung's disease is also known as multiple symmetrical lipomatosis, benign symmetrical lipomatosis, and Launois-Bensaude syndrome. The most commonly affected ones are men who drink alcohol in excessive amounts. The fat masses emerging in the course of the disease are painless but can lead to adverse repercussions. Patients may experience dysphagia, dysphonia, difficulty in breathing, and limited mobility of the neck. The reasons for the willingness to take up treatment are also often esthetic reasons. The disease is usually accompanied by numerous metabolic disorders. The etiology of the disease has not been sufficiently explained so far, which creates diagnostic and therapeutic difficulties. Currently used treatment is limited to surgical resection of the resulting lesions or liposuction. Unfortunately, the effectiveness of these activities is limited. Most patients experience recurrence after treatment. This paper discusses the essence of Madelung's disease, numerous aspects of etiology, the manner of diagnosis, and treatment based on current literature data.
\end{abstract}

Keywords: Madelung's disease, lipomatosis, multiple symmetrical

\section{Introduction}

Madelung's disease is also known as multiple symmetric lipomatosis (MSL), benign symmetric lipomatosis (BSL), and Launois-Bensaude syndrome. ${ }^{1-3}$ It is a rare disorder characterized by progressive, excessive, and symmetrical accumulation of adipose tissue in the subcutaneous layer, thus giving patients a peculiar appearance. ${ }^{1,2,4,5}$ Enzi $^{6}$ distinguishes two types of lipomatosis (Figure 1).

Another classification distinguishes three types of disease. ${ }^{7}$ The first one is characterized by the dominant cervical localization of excess fat; for the second type, the pseudoathletic appearance is considered characteristic, while in the third type - gynecological - changes are located in the area of the abdomen and hips. ${ }^{7,8}$ Regardless of the type of the disease, there is rarely any accumulation of fat around the larynx, mediastinum, and distal limbs. ${ }^{9,10}$ The described changes are painless, but they can lead to unfavorable, often-serious repercussions. ${ }^{4,10}$ Patients may develop dysphagia, dysphonia, and sleep apnea syndrome. There may be dif- 

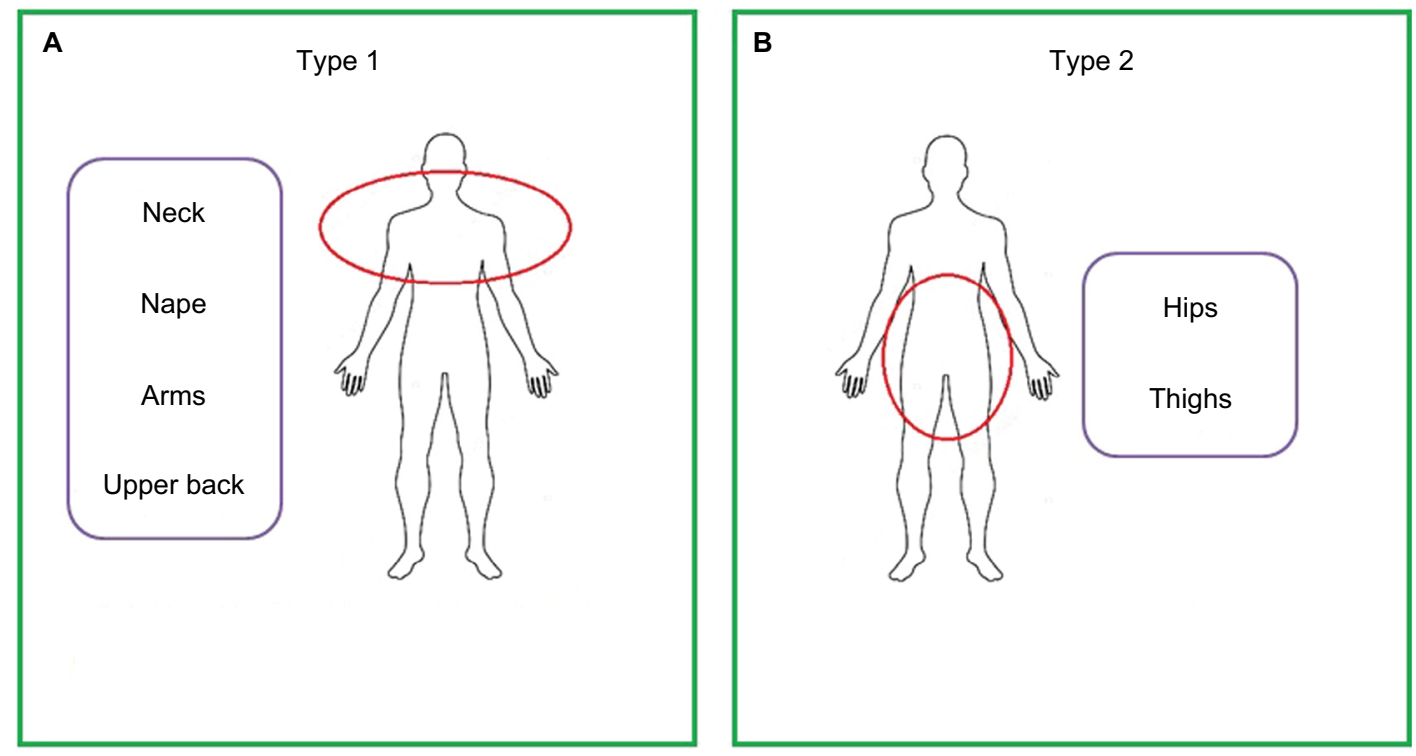

Figure I Madelung's disease: division based on the area of adipose tissue accumulation. Notes: (A) Type I: accumulating fatty tissue creates the so-called Madelung's collar and gives the figure a pseudoathletic look. (B) Type 2: the patient's silhouette is reminiscent of simple obesity.

ficulties in breathing and limited mobility of the neck. In patients, polyneuropathy is often observed. It is a generalized motor, sensory, and autonomic neuropathy not directly related to the location of fat mass. ${ }^{1,2,5,11}$ Another indirect consequence of the disease is the occurrence of depressive disorders in patients, resulting from the way they perceive themselves against the background of emerging changes in appearance. ${ }^{7}$

Madelung's disease affects men more often - the ratio of men to women varies from $15 / 1$ to $30 / 1$ - and is usually diagnosed between 30 and 70 years of age. ${ }^{1,412-14}$ Most cases of the disease are reported in the Mediterranean region the incidence in Italy has been reported to be as high as 1 in 25,000 men..$^{1,4,5,9,15}$ In contrast, in the Asian population, this disease is extremely rare. ${ }^{1,9}$ In the majority of cases, the disease is diagnosed in men in the 4th and 5th decades of life who consume chronically increased amounts of alcohol, especially in the form of red wine..$^{5,10,16}$ Moreover, $90 \%$ of patients also have secondary liver cirrhosis. ${ }^{4,7}$ The most frequently diagnosed phenotype of the disease is of type 1, with a dominant fat deposit around the neck..$^{13,14,16}$

\section{Case report}

A 53-year-old man with upper body deformity, a strongly limited neck mobility, and weakening of the muscles of the shoulders and upper limbs came to the clinic. The patient had numerous, clearly limited, round, subcutaneous fat masses protruding above the body surface. These changes were arranged symmetrically on the neck, shoulders, upper torso, and the proximal part of the upper limbs. The patient had a pseudoathletic look, and the fatty tissue around the neck formed a typical "Madelung's collar". In an interview, the patient admitted that he had been abusing alcohol for 39 years. However, 9 months earlier, he was subjected to tests, on the basis of which he was diagnosed with alcohol-induced decompensated liver cirrhosis. At the time of admission, the calculated patient body mass index (BMI) was $24.7 \mathrm{~kg} /$ $\mathrm{m}^{2}$, with a waist circumference of $81 \mathrm{~cm}$. The patient was not diagnosed with overweight or obesity, and biochemical tests did not show disturbances in either lipid or coagulation parameters. However, it was noticed that gynecomastia, limb muscle abnormalities, and hepatosplenomegaly occurred in the examined man. Laboratory tests showed the following disorders: hypochromic anemia (hemoglobin $11 \mathrm{~g} / \mathrm{dL}$, red blood cell count $\left.3,900 \times 10^{6} / \mathrm{mL}\right)$ neutropenia $\left(1.3 \times 10^{3} /\right.$ $\mu \mathrm{L})$, thrombocytopenia $\left(100 \times 10^{3} / \mu \mathrm{L}\right)$, and slightly elevated hepatic (AST: $130 \mathrm{U} / \mathrm{L}$ and ALT: $90 \mathrm{U} / \mathrm{L}$ ) and pancreatic (lipase: $170 \mathrm{U} / \mathrm{L}$ and amylase: $140 \mathrm{U} / \mathrm{L}$ ) enzymes. The pictures of the patient with the described deformities are shown in Figure 2. 


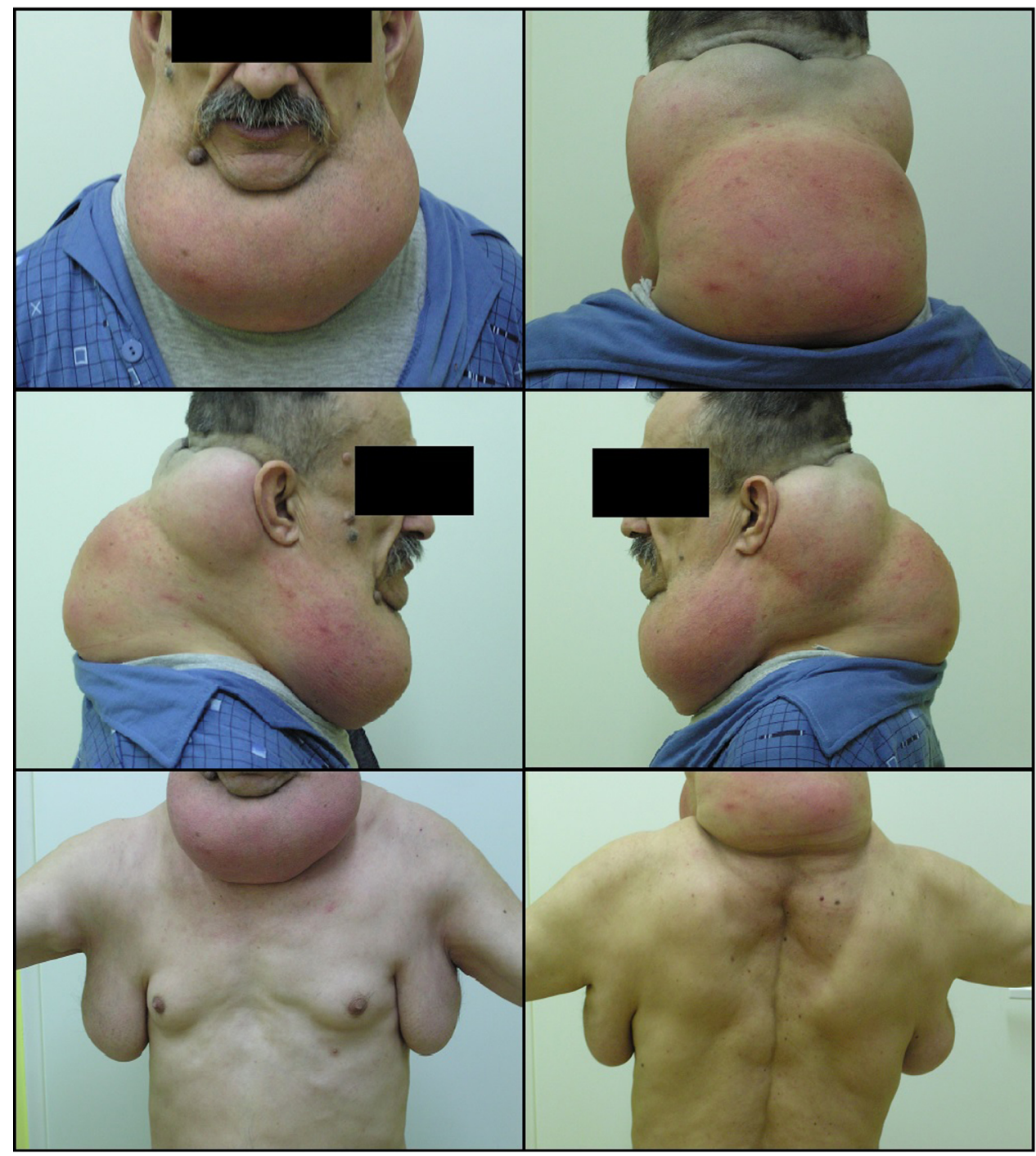

Figure 2 Patient with Madelung's disease.

Endocrine workup showed abnormalities. Hyperestrogenism was confirmed with normal testosterone levels and decreased dehydroepiandrosterone sulfate (DHEA-S) levels. The patient was diagnosed with type 2 diabetes based on an oral glucose tolerance test. The results of additionally tested parameters, ie, insulin, C-peptide, and thyroid hormones, were within the reference values. Finally, the patient was diagnosed with a rare disorder of fat proliferation, which is called Madelung's disease. It was found that the disease phenotype presented by the man corresponds to type 1 according to the Enzi ${ }^{6}$ classification. The patient underwent computed tomography to visualize the structure of lesions and determine their location. The study also allowed to determine the degree of penetration of fat masses to deeper structures. Based on the history, clinical status, and examinations, it was decided to qualify the patient for surgical treatment. Surgical resection of excess adipose tissue was performed. In the postoperative period, there were no complications, and the patient's well-being was assessed as good. The patient remained under observation for 3 years without recurrence.

\section{Discussion}

Madelung's disease was first described in the year 1846 by Brodie $^{17}$ and then, in 1888, it was also described by Madelung. ${ }^{18}$ In 1898, French physicians Launois and Bensaude ${ }^{19}$ published the characteristics of this disease based on a group of 65 patients. According to the data for 2012, there are $>270$ cases of Madelung's disease in the literature. ${ }^{20}$ Chen et a ${ }^{21}$ summarized the clinical data of the cases described in the literature in the years 2000-2015 (Table 1). 
Table I Summary of the clinical data of 4 I literature sources of Madelung's disease from 2000 to 2015 , with complete material on treatment

\begin{tabular}{|l|l|}
\hline Number of cases & $\mathbf{1 0 6}$ \\
\hline Ethnic group & $\begin{array}{l}\text { Mostly } \\
\text { Mediterranean } \\
\text { origin }\end{array}$ \\
\hline Type of disease & $\begin{array}{l}\text { Mostly type I } \\
\text { (Enzi's classification) }\end{array}$ \\
\hline Treatment & \\
• Lipectomy & 95 \\
- Liposuction & 18 \\
- Other & 9 \\
\hline Treatment effect & \\
• Relapse (follow-up times) & 7 \\
• No relapse (follow-up times) & 44 \\
• Unknown & 55 \\
\hline
\end{tabular}

Madelung's disease remains a disorder of unexplained etiology and unclear pathogenesis. ${ }^{1}$ The distribution and type of adipocytes in patients with Madelung's disease resemble the brown adipose tissue (BAT) of infants. ${ }^{5,8,11}$ In patients, instead of increasing the size of existing fat cells, new ones appear. ${ }^{7}$ The results of a long-term study, in which 7 patients were observed over a period of 12 years, confirm the hypothesis that lipomas formed in the course of the disease are the result of mitochondrial disorders of BAT. ${ }^{12,22}$ Other authors also indicate that disorders of proliferation and differentiation of human BAT cells underlie the described disease. . $^{1,410}$

There are studies that reveal the relationship of Madelung's disease with diabetes, hyperlipidemia, hypertension, or liver disease. However, the influence of excess body fat on the metabolism of the body is still under discussion. ${ }^{1}$ Regardless of this, people with Madelung's disease often have coexistence of other disorders (Figure 3).

This is confirmed by the described case, in which the patient shows the occurrence of diabetes, hepatosplenomegaly, cirrhosis, and gynecomastia simultaneously with Madelung's disease. Apart from these disorders, sensory, motor, and autonomic polyneuropathy is additionally observed in approximately $85 \%$ of patients with Madelung's disease. ${ }^{9,12}$

One of the main factors for the development of this disease is alcohol abuse. Alcohol adversely affects the enzymatic processes in the mitochondria and disrupts adrenergic lipolysis - it can be a cofactor inducing a change in the number and function of $\beta$-adrenergic receptors. It is likely that alcohol abuse is the cause of uncontrolled accumulation of adipose tissue in various parts of the body. ${ }^{3,7,11}$ The described case also presents a long history of consuming excessive amounts of alcohol. The literature describes only a few cases of Madelung's disease without alcohol in the patient's diet. ${ }^{24}$ Other possible risk factors include nicotine abuse, metabolic disorders, and malignant tumors. ${ }^{4}$

Most likely, there is genetic predisposition based on matrilineal inheritance. ${ }^{1,2,4,9}$ It is recognized that the disease may be inherited in an autosomal dominant manner with variable mitochondrial DNA penetration. ${ }^{3,8,10,12}$

Archeological discoveries suggest that there may be an inherited tendency for Madelung's disease - perpetuated for centuries - among the population of people living in the Mediterranean. This may be evidenced by a statue known as the Capestrano warrior from the 6th century BC and discovered in Italy. It presents an impressive figure with a unique somatic aspect, characterized by large hips and broad shoulders. The silhouette of the Capestrano warrior is very similar to the appearance of a patient with Madelung's disease. This convergence may be accidental and may also indicate the genetic basis of the occurrence of this disease. ${ }^{25}$

A case report of a familial Madelung disease in siblings, ie, a 41-year-old woman and her 36-year-old brother, has been described in the literature. In molecular studies, the existence of mutations in the lipase-encoding gene has been demonstrated in both patients. ${ }^{26}$ In the studies carried out so far, Madelung's disease is associated with point mutations in mitochondrial DNA, whose genes are important in the regulation of lipolytic pathway processes. ${ }^{1,22}$ A relationship between the described disease and the occurrence of myoclonic epilepsy and shred of red fibers has also been established. ${ }^{1}$ In the muscles of patients with Madelung's disease, the occurrence of radiant red fibers is often found, which indicates mitochondrial dysfunction with reduced cytochrome c oxidase activity. ${ }^{2,10,11}$

The local defect of catecholamine-induced lipolysis, adrenal adrenoceptor defects, and the reduction of inducible nitric oxide synthase (iNOS) are potential causes of Madelung's disease. The implication of these processes is increased adipogenesis. ${ }^{1,3,9,10}$ In addition, recently conducted molecular studies using next-generation sequencing have also demonstrated the existence of genetically diverse genes in patients with Madelung's disease compared to healthy controls. ${ }^{1}$

The diagnosis of Madelung's disease is based on history, clinical appearance of the patient, and the results of imaging examinations (Figure 4).

The disease should be differentiated from morbid obesity, Cushing's syndrome, salivary gland disease, lymphoma, Froelich's syndrome, angiolipomatosis, myxoid liposarcoma, encapsulated lipomas, neurofibromatosis, and lipomatosis in 


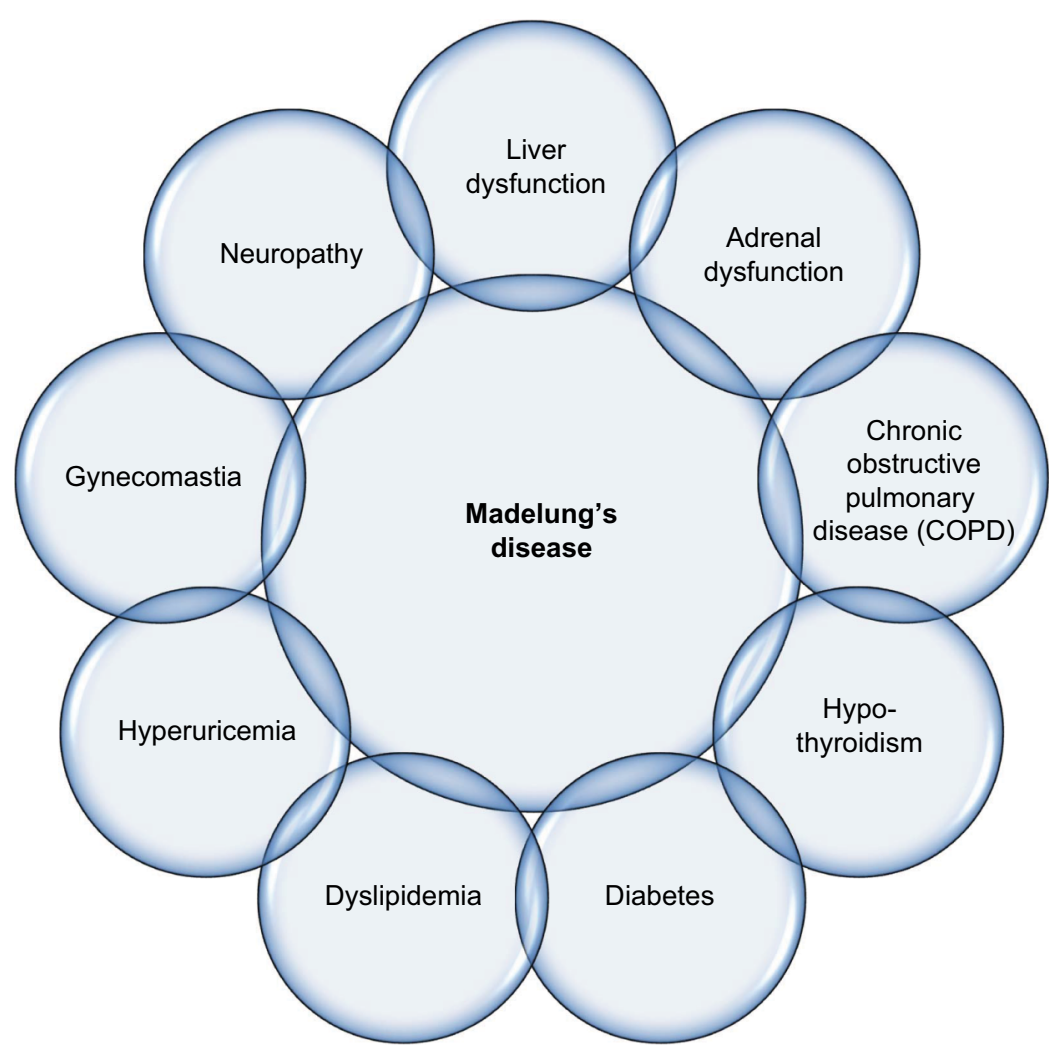

Figure 3 Disorders often co-occurring with Madelung's disease. Notes: Data from these studies. ${ }^{2,4,9,16,23}$

\section{History and physical examination of the patient}

\section{Ultrasonography}

- Ultrasonography enables fine needle biopsy

\section{Computed tomography}

- Computed tomography is the method of choice for the diagnosis, preoperative staging, and postoperative follow-up of patients with Madelung's disease

\section{Additional tests}

\section{- X-Ray}

- Magnetic resonance

- Laboratory tests

Figure 4 Examinations used in the diagnosis of Madelung's disease.

Notes: Data from these studies. 1,9,13,20

patients infected with $\mathrm{HIV}^{4,12}$ The disease progresses rapidly in the first few years, after which the progression usually slows down or the size of the created adipose tissue mass stabilizes. ${ }^{3,8}$

The therapeutic approach in Madelung's disease is reduced to palliative treatment, which includes surgical resection of the adipose tissue, liposuction, or injection lipolysis. ${ }^{1-4}$ It seems reasonable to correct metabolic disorders and overweight. ${ }^{3}$ Alcohol withdrawal and weight loss may be helpful in controlling the disease, but they do not guarantee the inhibition of its progression. ${ }^{1,3,16}$ Surgical procedures are time consuming - often divided into several stages - due to the volume of fat mass, as well as their spread and infiltration into neighboring tissues. ${ }^{3,4}$ Conventional surgery is recommended, especially 
in heavily vascularized and innervated regions, as well as in violation of patency of the airways and esophagus. ${ }^{3,13}$ The efficacy of surgical treatment or liposuction is limited. ${ }^{1,14}$ It is estimated that nearly $63 \%$ of patients experience recurrence after surgical resection of adipose tissue, while in the case of liposuction, up to $95 \%$ of patients experience recurrence. . $^{1,8,16}$ After injection lipolysis, fibrosis and adhesions form. This impedes surgical treatment or liposuction in the recurrence of lesions. ${ }^{11}$ Despite frequent relapses, functional and cosmetic effects obtained in surgical treatment can be satisfactory. ${ }^{4}$ Unfortunately, no effective pharmacotherapy has been developed so far in the treatment of Madelung's disease. The use of salbutamol for adrenergically stimulating lipolysis and thus inhibiting the progression of the disease in larger clinical trials did not show the expected effect. . $^{8,12}$

\section{Conclusion}

Madelung's disease - apart from the essential excessive accumulation of adipose tissue - is characterized by the occurrence of numerous metabolic disorders. In the literature, the authors describe the coexistence, among others, of carbohydrate, lipid, or hormonal disorders along with the disorder in question. Diagnosis of diabetes and abnormal levels of sex hormones in the presented case are the confirmation.

The described case also confirms the role of excessive alcohol consumption in the pathogenesis of the disease. In contrast, impaired liver function - occurring in the majority of patients with Madelung's disease - may have a greater impact on the metabolic abnormalities than the pathology of adipose tissue alone.

Madelung's disease is rarely diagnosed, which may not necessarily be due to the low incidence of this disease but may be associated with the diagnostic and therapeutic difficulties, as well as with the availability of few literature reports, often related to individual cases.

\section{Consent for publication}

The patient provided written informed consent for publication of the clinical details and images.

\section{Disclosure}

The authors report no conflicts of interest in this work.

\section{References}

1. Chen K, Wang L, Yang W, Wang C, Hu G, Mo Z. Profiling of differentially expressed genes in adipose tissues of multiple symmetric lipomatosis. Mol Med Rep. 2017;16(5):6570-6579.
2. Nisi G, Sisti A. Images in clinical medicine. Madelung's disease. $N$ Engl J Med. 2016;374(6):572.

3. El Ouahabi H, Doubi S, Lahlou K, Boujraf S, Ajdi F. Launois-Bensaude syndrome: a benign symmetric lipomatosis without alcohol association. Ann Afr Med. 2017;16(1):33-34.

4. Ardeleanu V, Chicos S, Georgescu C, Tutunaru D. Multiple benign symmetric lipomatosis - a differential diagnosis of obesity. Chirurgia. 2013;108(4):580-583.

5. Prahlow SP, Kosciuk P, Prahlow JA. Multiple symmetric lipomatosis. J Forensic Sci. 2018;63(1):312-315.

6. Enzi G. Multiple symmetric lipomatosis: an updated clinical report. Medicine. 1984;63(1):56-64.

7. Zielińska-Kaźmierska B, Lewicki M, Manowska B. Madelung disease. Advances in Dermatology and Allergology. 2015;5:400-403.

8. Jang JH, Lee A, Han SA, Ryu JK, Song JY, Lipomatosis MS. Multiple Symmetric Lipomatosis (Madelung's Disease) Presenting as Bilateral Huge Gynecomastia. J Breast Cancer. 2014;17(4):397-400.

9. Yeh NC, Yang CY, Chou CW, Yen FC, Lee SY, Tien KJ. Madelung's disease. J Clin Endocrinol Metab. 2012;97(9):3012-3013.

10. Enzi G, Busetto L, Sergi G, et al. Multiple symmetric lipomatosis: a rare disease and its possible links to brown adipose tissue. Nutr Metab Cardiovasc Dis. 2015;25(4):347-353.

11. Wollina U, Heinig B. Madelung's Disease - Case Series and Treatment by Tumescent Liposuction or Lipectomy. Open Access Maced J Med Sci. 2017;5(4):427-431.

12. Esposito AC, Munhoz T, Abbade LP, Miot HA. Do you know this syndrome? Type 2 benign symmetric lipomatosis (Launois-Bensaude). $A n$ Bras Dermatol. 2016;91(6):840-841.

13. Chen HW, Chen HW, Chen HL, Lai CC. Madelung Disease. Am J Med Sci. 2016;352(6):654.

14. Pinto CI, Carvalho PJ, Correia MM. Madelung's Disease: Revision of 59 Surgical Cases. Aesthetic Plast Surg. 2017;41(2):359-368.

15. Landis MS, Etemad-Rezai R, Shetty K, Goldszmidt M. Case 143: Madelung disease. Radiology. 2009;250(3):951-954.

16. Brea-García B, Cameselle-Teijeiro J, Couto-González I, Taboada-Suárez A, González-Álvarez E. Madelung's disease: comorbidities, fatty mass distribution, and response to treatment of 22 patients. Aesthetic Plast Surg. 2013;37(2):409-416.

17. Brodie BC. Clinical Lectures on Surgery delivered at St. George's Hospital. Am J Med Sci. 1846;11(22):437.

18. Madelung OW. Ueber den Fetthals. Arch Klin Chir. 1888;37:106-130.

19. Launois PE, Bensaude R. De l'adenolipomatose symetriqe. Bull Mem Soc Med Hop Paris. 1898;1:298.

20. Persici Rodrigues L. Lima Araujo Melo E. Madelung's disease: a case report and literature review. Radiol Bras. 2012;45:2.

21. Chen CY, Fang QQ, Wang XF, et al. Madelung's Disease: Lipectomy or Liposuction? Biomed Res Int. 2018;2018(21):3975974.

22. Plummer C, Spring PJ, Marotta R, et al. Multiple Symmetrical Lipomatosis - a mitochondrial disorder of brown fat. Mitochondrion. 2013;13(4):269-276.

23. Enzi G, Busetto L, Ceschin E, Coin A, Digito M, Pigozzo S. Multiple symmetric lipomatosis: clinical aspects and outcome in a long-term longitudinal study. Int $J$ Obes Relat Metab Disord. 2002;26(2):253-261.

24. Bergler-Czop B, Wcisło-Dziadecka D, Brzezińska-Wcisło L. Madelung's disease in a patient with chronic renal insufficiency: a case report and review of literature. Postepy Dermatol Alergol. 2014;31:121-124.

25. Feliciani C, Amerio P. Images in clinical medicine. Madelung's disease: inherited from an ancient Mediterranean population? N Engl J Med. 1999;340(19): 1481.

26. Zolotov S, Xing C, Mahamid R, Shalata A, Sheikh-Ahmad M, Garg A. Homozygous LIPE mutation in siblings with multiple symmetric lipomatosis, partial lipodystrophy, and myopathy. Am J Med Genet A. 2017;173(1):190-194. 
Diabetes, Metabolic Syndrome and Obesity: Targets and Therapy

Diabetes, Metabolic Syndrome and Obesity: Targets and Therapy is an international, peer-reviewed open-access journal committed to the rapid publication of the latest laboratory and clinical findings in the fields of diabetes, metabolic syndrome and obesity research Original research, review, case reports, hypothesis formation, expert opinion and commentaries are all considered for publication. The manuscript management system is completely online and includes a very quick and fair peer-review system, which is all easy to use. Visit http://www.dovepress.com/testimonials.php to read real quotes from published authors.

Submit your manuscript here: https://www.dovepress.com/diabetes-metabolic-syndrome-and-obesity-targets-and-therapy-journal 